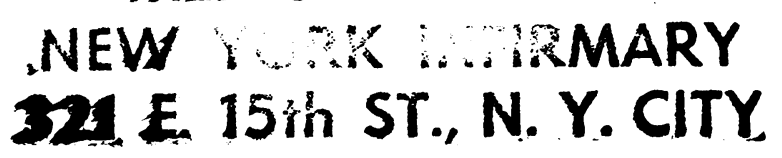

Journal of Neurology, Neurosurgery, and Psychiatry, 1980, 43, 469-474

\title{
Electrophysiological cross section of the motor unit
}

\author{
E STÅLBERG AND L ANTONI \\ From the Department of Clinical Neurophysiology, University Hospital, Uppsala
}

SUMMARY A new technique to investigate the motor unit organisation in man described. By moving an EMG electrode through the motor unit a continuous scan or "electrophysiological cross section" is obtained. Computer processing methods are used for analysis and display. Examples are shown from healthy subjects and from patients with neuromuscular diseases.

Electromyographic recordings are conventionally performed by means of a concentric needle EMG electrode. The signal obtained from one motor unit represents the temporal and spatial summation of action potentials from a number of its individual muscle fibres. The contribution to the motor unit potential (MUP) from one muscle fibre depends on the distance from the fibre to the recording electrode. With increasing distance the amplitude decreases and the signal contains relatively more slow frequency components. The shape of the MUP also depends on the volume conduction properties of the muscle, the filter characteristics of the tissue, the recording electrode and the amplifier system. It has been estimated that the spike component of the MUP is derived mainly from about 10 muscle fibres in the normal muscle. ${ }^{1}$ This corresponds to an uptake radius of about $1 \mathrm{~mm}$. Considering that a normal motor unit has at least 200 to 300 muscle fibres $^{2}$ distributed throughout a territory of 5-10 $\mathrm{mm}$ diameter $^{34}$ it is clear that the MUP only represents a minor part of all fibres in a motor unit, often of the order of $5 \%$. The shape of the recorded MUP is therefore dependent on the relation between the recording electrode and the muscle fibres within one motor unit. From routine work with EMG it is known that the shape of a MUP may change considerably with slight needle movements, contributing to an uncertainty in quantifying the EMG. New information about the motor unit can be obtained by means of a scanning procedure. One method of studying the motor unit territory was described earlier ${ }^{3}$ based on 14-lead multielectrode recordings. In the present technique a concentric needle

Address for reprint requests: Dr E Stålberg, University Hospital, S 75014 Uppsala, Sweden.

Accepted 3 January 1980 electrode is moved through the territory of the motor unit. This gives the continuous shape of the electrical field within a corridor of the motor unit measuring $1 \mathrm{~mm}$ in radius for the spike components but considerably more for the slower components. The method is described and some results are given to illustrate the potential value of the method.

\section{Method}

A single fibre EMG electrode is inserted into the voluntarily activated muscle and action potentials from a muscle fibre belonging to a low threshold motor unit are recorded. This recording is kept constant throughout the procedure. The single fibre action potential is used to trigger the oscilloscope sweep. Next a concentric needle electrode is inserted $10-20 \mathrm{~mm}$ from the single fibre EMG electrode. A position is sought where a MUP synchronous to the triggering single fibre action potential, that is appearing at a constant position along the oscilloscope sweep, is obtained. By means of a signal delay line this MUP is positioned in the middle of a $15 \mathrm{~ms}$ sweep of the oscilloscope. Before the scanning starts the electrode is now carefully pushed $10 \mathrm{~mm}$ deeper into the muscle. During a slight voluntary activation giving a low constant firing rate of the motor unit the concentric electrode is pulled continuously for a distance of $20 \mathrm{~mm}$ through the muscle, as determined by markings on the electrode shaft (fig 1). All the MUP's (typically 150-400) are stored on analogue tape during the scanning procedure.

\section{Analysis}

The result is presented in various graphical displays produced by means of a computer, either a PDP $11 / 40$ or a microprocessor LSI 11. The 


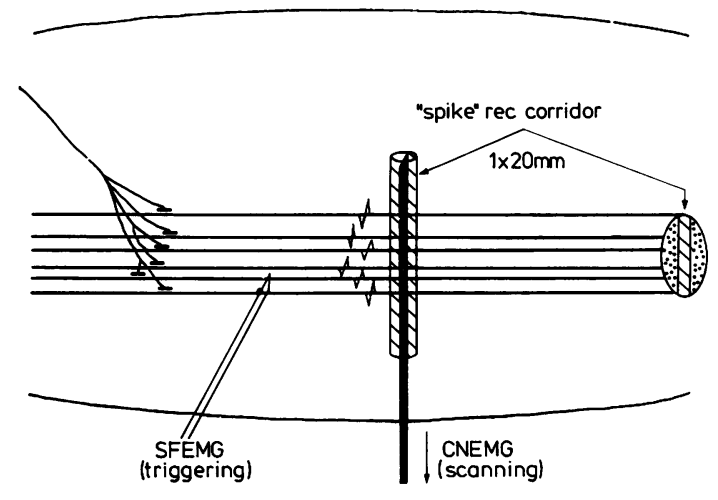

Fig 1 Schematic explanation of the recording procedure. A single fibre EMG electrode is used to record action potentials from one muscle fibre, triggering the oscilloscope. The concentric needle EMG electrode (CNEMG) is pulled through the muscle. Motor unit potentials synchronous to the triggering action potential are stored. The uptake area for spike components, the "spike recording corridor" is $1 \times 20 \mathrm{~mm}$.

construction of the electrophysiological cross section is done in two steps since the motor unit firing at a rate of about 10 impulses/sec does not allow real time processing between the discharges. The first step is data acquisition and storage on disc. The second part is to construct a picture on the electrostatic printer/plotter.

The digitising of the motor unit potential is done by means of a 12 bit A/D converter. The trigger signal from the single fibre EMG electrode to which the motor unit signal is locked is used to initiate the A/D conversion. The sampling interval is usually set to $30 \mu \mathrm{s}$ and the sweep length is 512 points equivalent to $15.36 \mathrm{~ms}$. Each digitised sequence is stored in a random access file. This procedure is repeated for each discharge during each $20 \mathrm{~mm}$ needle movement through the muscle.

The next step is to plot each recorded motor unit potential on the plotter using a special purpose program, 3 DIM. Each new MUP is displaced a small distance along the $Y$ and $X$ direction on the plot (Y-direction downwards, $\mathrm{X}$-direction across the paper). If the new MUP is hiding any of previous ones plotted the hidden parts are erased from the plot giving a three dimensional impression. The program is highly optimised for this kind of plot and the time to process a plot of 250 discharges with moderate hidden line elimination is four minutes.

In another display the amplitudes are converted into a greyscale with 16 tones from black to white for signals going from negative to positive. The dynamics and absolute values for the correlation between amplitude and tone can be optionally adjusted. In another display colour is used instead of grey tones. In this case the resolution is $\mathbf{2 5 6}$ different colour tones.

\section{Results}

Recordings are obtained from the tibialis anterior and biceps brachii muscles in healthy subjects and from patients with different neuromuscular disorders. In this report some principle findings will be described. In the recording from the normal muscle the "spiky responses" from one motor unit are restricted to 5 to $10 \mathrm{~mm}$ of the corridor but the slow components are usually recorded over a larger distance.

When the electrode is moved some hundred microns the shape of the individual MUP changes considerably and has quite different shapes when the steps exceed $1 \mathrm{~mm}$ (fig 2).

The muscle fibres in the normal motor unit seem to be mainly evenly distributed. Usually the motor unit potentials are concentrated within one area of the recording corridor. The electrical "front" is sometimes perpendicular to the estimated fibre direction but does sometimes appear with an angle to the fibre direction, in dicating that the potentials reach the electrode earlier in one recording area than in the other. This is either due to a skew recording corridor through the muscle, although attempts are made to have it perpendicular, or reflect the anatomical arrangement of the motor end-plates in the muscle.

In the abnormal muscle the motor unit cross section differs, sometimes considerably, from the normal. In cases with a neurogenic lesion the total duration of the MUP is increased, the width of the front is broader, the amplitude of the MUP is increased and therefore the action potential is recorded over a larger distance due to volume conduction. The anatomical length over which the spiky part of the MUP is recorded is not necessarily increased. Sometimes the MUP's are seen concentrated in two different areas within the corridor probably indicating an abnormal concentration of fibres in these two areas (fig 3).

In muscular dystrophies or acute polymyositis the number of fibres in the motor unit is reduced and the MUP is polyphasic and of short duration. Responses from the same motor unit may be obtained from different and separated areas along 


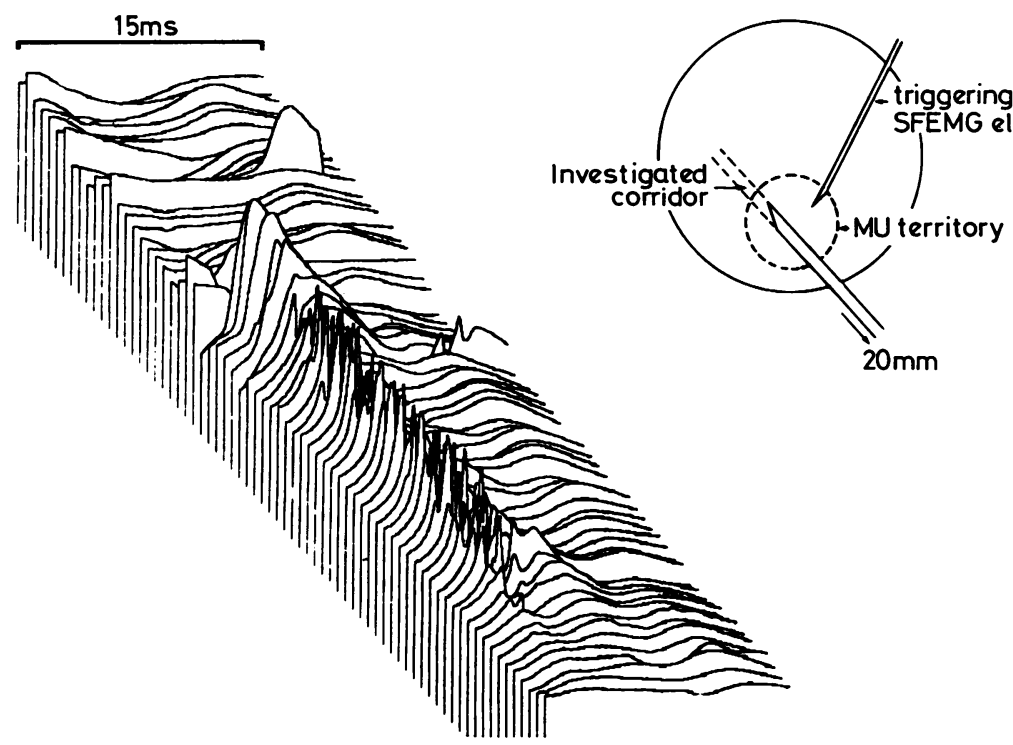

Fig 2 An example of recording from a normal tibialis anterior muscle. Note the great variation in shape of MUPs for different electrode positions.
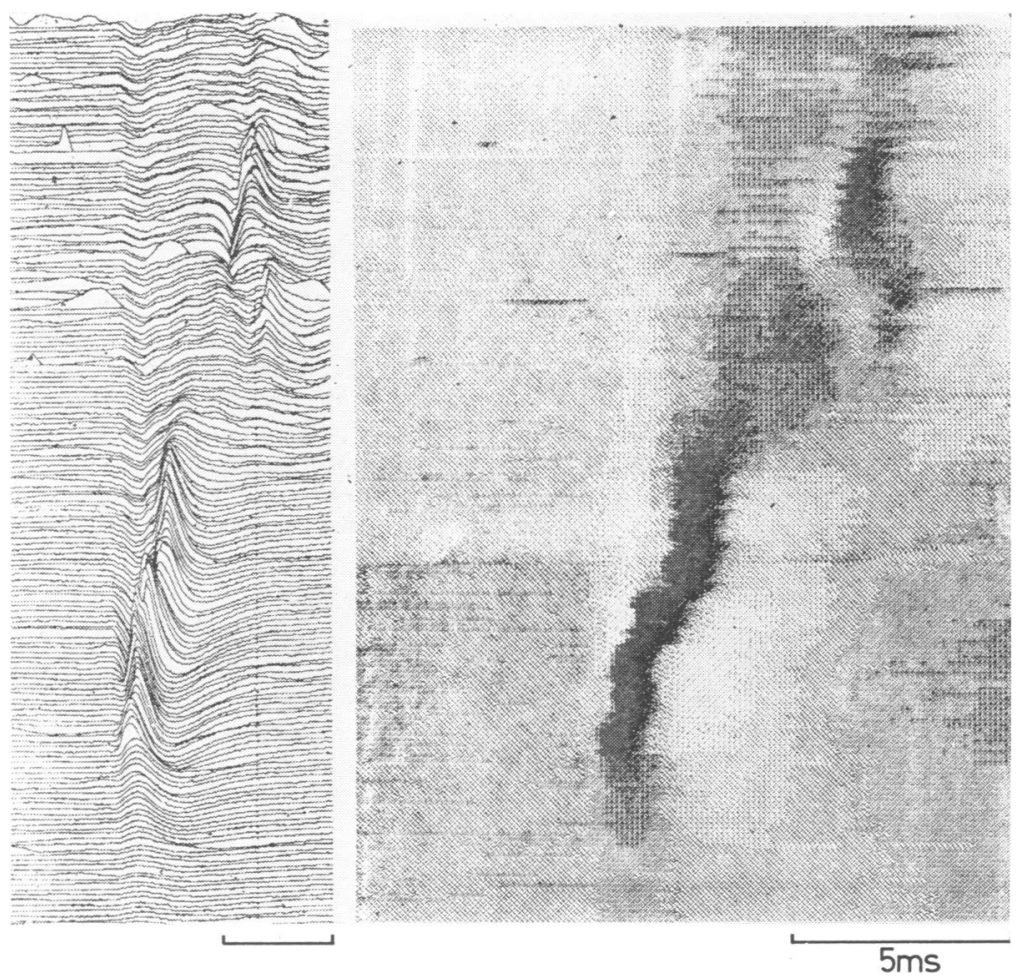

Fig 3 Recording from $a$ tibialis anterior muscle in a patient with history of $L 5$ syndrome. To the left are the MUPs obtained with continuous needle movements, to the right a greyscale plot where amplitudes are converted to black-white intensities. Note the extended area over which activity is recorded compared to normal (fig 2) due to the strong electrical field after reinnervation. The fibres of the motor unit are in this recording concentrated to two areas. the corridor, sometimes with distinctly separated arrival times to the recording electrode. The recording may show areas within the territory without any electrical activity from the motor unit under study (figs 4 and 5).

In many scannings a special phenomenon is seen (fig 6). With the needle in the deepest position in the muscle a low amplitude initially 


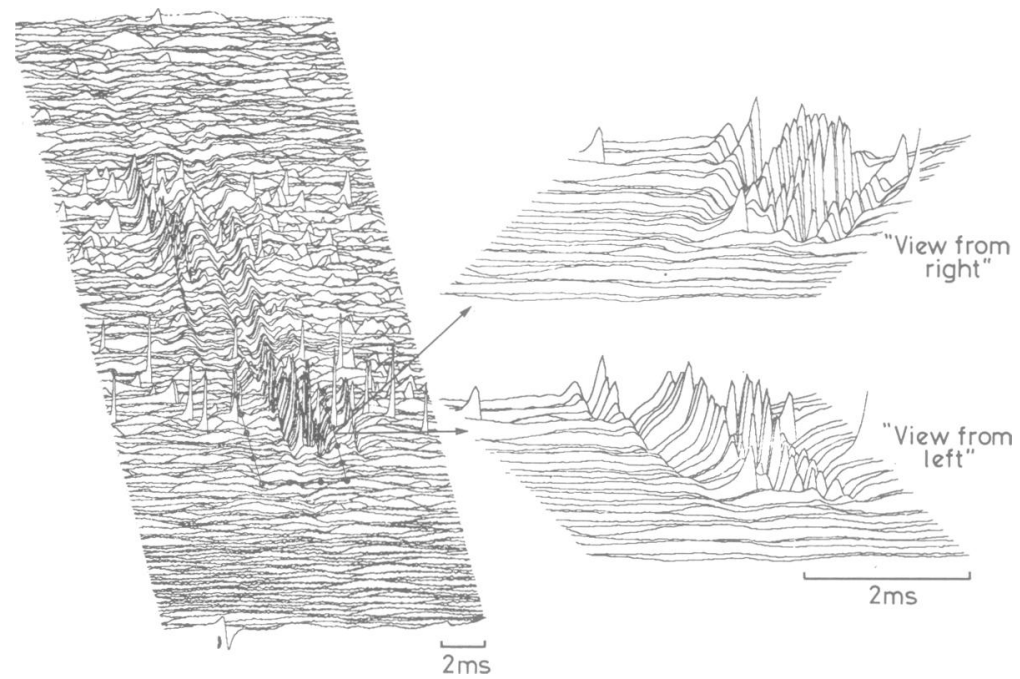

Fig 4 Recording from a tibialis anterior muscle of a patient with muscular dystrophy. The motor unit is disintegrated. Individual MUPs are polyphasic. The "blow up" shows such an area from two "angles". The random spikes in the plot are generated by other motor units.
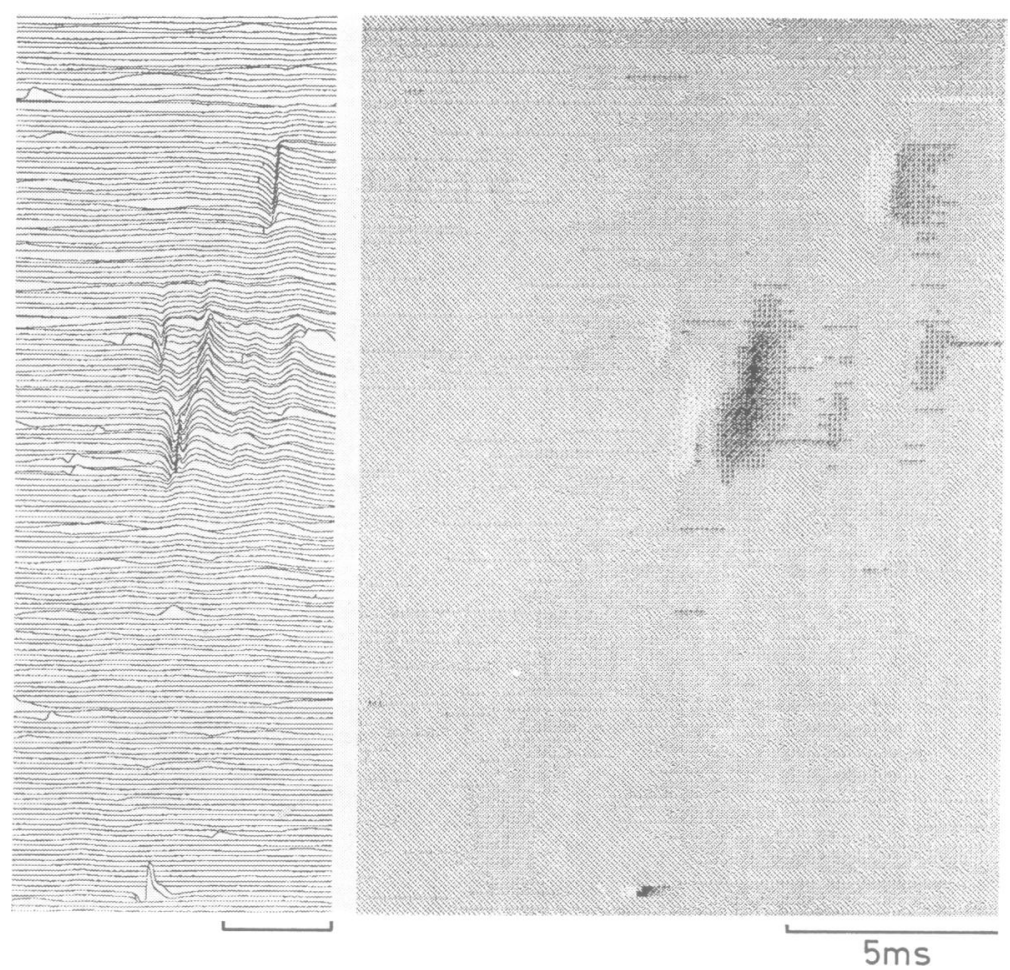

Fig 5 Recording from tibialis anterior muscle of a patient with polymyositis. The motor unit is disintegrated.

$A$ silent area is seen between the two regions with activity. positive going deflection without spike components, a "trough", is obtained. This is unchanged in amplitude during the scanning until the electrode is recording a normal MUP with initially negative components. These two responses have the same delay. When the needle is pulled further the MUP disappears and the positive trough is not seen any longer. If the scanning shows two separate areas of muscle fibre activity within the motor unit each of them may be "announced" during the earlier part of the scanning by a trough. 

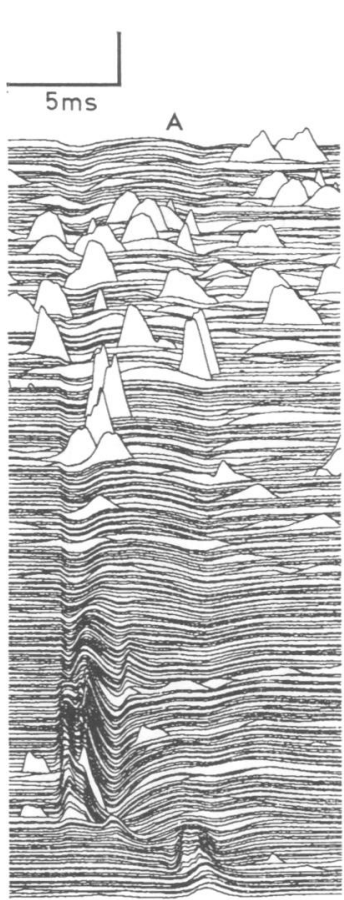

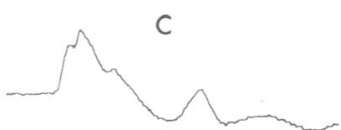

B

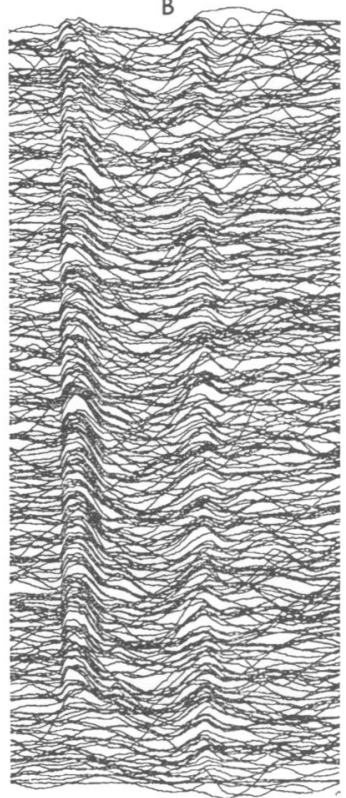

Fig 6 Scanning in a normal tibialis anterior muscle. A Scanning with concentric needle electrode, $B$ Simultaneous recording between needle shaft and a separate remote reference electrode, $C$ Averaged recording from $B$, with the needle at its deepest position (top of the plot).

This corresponds to the "Macro EMG". ${ }^{6}$ The picture demonstrates that the cannula also is recording activity from the motor unit. This is seen as a

"trough" in the scanning $(A)$ which "announces" the fibres before they are recorded by the tip electrode. $Y$ Calibration: for $A=200 \mu \mathrm{V}$, for $B=100 \mu \mathrm{V}$, for $C=50 \mu V$.

The explanation is the following. With the needle in its deepest position the cannula, normally acting as reference, is recording activity from the muscle fibres not yet heard by the tip electrode. This corresponds to the Macro $\mathrm{EMG}^{5}{ }^{6}$ where in this case the tip electrode is acting as reference (therefore the polarity is opposite to that of the MUP). The trough is unchanged until the needle is pulled to a position where the tip electrode is recording activity from these fibres and is not seen when the electrode is pulled out of the motor unit. This cannula response is further demonstrated when recording is made between the cannula and a separate reference electrode placed subcutaneously. The response is unchanged during the whole scanning until the tip has passed the active fibres. This trough is naturally not seen if the scanning EMG is made with insulated monopolar electrodes.

The trough is usually of higher amplitude when the recorded MUP is of high amplitude, for example in neurogenic diseases and small when the MUP is small (figs 3 and 5). This correlation is however not always seen since the cannula recording (Macro EMG) is recording over a larger area than the tip and can therefore detect parts of the motor unit outside the recording corridor for the tip electrode. In conventional EMG recordings these troughs can sometimes be seen as early or late components in the MUP and can be included in duration measurements. One should be aware of the fact that this activity may be recorded by the cannula, the reference.

\section{Discussion}

The present method for the study of the motor unit gives some additional information about the organisation of its individual muscle fibres and about the propagation of the action potentials within the motor unit. The recording still does not represent the whole motor unit but gives information about a cross section of the motor unit. The total area over which a motor unit can be recorded is a minimal value of the territory since the recording corridor may well be at the periphery of a motor unit. The territory of the motor unit as seen with this technique in the normal biceps brachii muscle mainly corresponds to previously published figures. ${ }^{34}$

The results amplify earlier knowledge that the so-called motor unit potential represents only a minor part of the motor unit and that large variations in MUP shape can be obtained within one motor unit. The question of a defined recording position in clinical EMG is still not solved.

By means of this method it seems possible to get further information about the pathological motor unit, its territory and the arrangement of muscle fibres within the territory. It includes the conventional MUP parameters but adds information about the temporal and spatial distribution of action potentials within different parts of the motor unit. It is also possible to obtain information about "silent areas" within the motor unit, information which is not achieved quantitatively in the conventional way of performing electromyography.

This investigation was supported by the Swedish Medical Research Council (Grant No. 135). 


\section{References}

1 Thiele B, Boehle A. Number of single muscle fibre action potentials contributing to the motor unit potential. 5th International Congress of EMG, Rochester, Minnesota, 1975.

Stålterg E, Gath I. In situ measurement of the innervation ratio of motor units in human muscles. In press in IEEE Transactions on Biomedical Engineering.

3 Buchthal F, Guld Ch, Rosenfalck P. Multielec- trode study of the territory of a motor unit. Acta Physiol Scand 1957; 39:83-103.

4 Stålberg E, Schwartz MS, Thiele B, Schiller HH. The normal motor unit in man. J Neurol Sci 1976; 27:291-301.

5 Stalberg E. The Macro EMG. 6th International congress of EMG (Ed. Persson A). Abstracts. Acta Neurol Scand 1979; Suppl 73: 190.

6 Stålberg E. Macro EMG. A new recording technique. J Neurol Neurosurg Psychiatry 1980; 43: 475-82. 